\title{
The application value of metagenomic next-generation sequencing in children with invasive pneumococcal disease
}

\author{
Fang Guo $^{1 \wedge}$, Lei Kang ${ }^{2}$, Meixian $\mathrm{Xu}^{2}$ \\ ${ }^{1}$ Department of Infection, Hebei Children's Hospital, Affiliated to Hebei Medical University, Shijiazhuang, China; ${ }^{2}$ Department of Pediatric \\ Intensive Care Unit, Hebei Children's Hospital, Affiliated to Hebei Medical University, Shijiazhuang, China \\ Contributions: (I) Conception and design: F Guo; (II) Administrative support: M Xu; (III) Provision of study materials or patients: F Guo, L Kang; \\ (IV) Collection and assembly of data: F Guo, L Kang; (V) Data analysis and interpretation: L Kang; (VI) Manuscript writing: All authors; (VII) Final \\ approval of manuscript: All authors. \\ Correspondence to: Meixian Xu. Department of Pediatric Intensive Care Unit, Hebei Children's Hospital, Affiliated to Hebei Medical University, 133 \\ Jianhua South Street, Shijiazhuang 050031, China. Email: 13833185617@163.com.
}

Background: A retrospective analysis was conducted to explore the sensitivity and specificity of metagenomic next-generation sequencing (mNGS) in blood, cerebrospinal fluid, and pleural effusion samples in children with invasive pneumococcal disease (IPD), and the impact of detection timing on prognosis and cost.

Methods: Children with IPD admitted to Hebei Children's Hospital from 1 January 2017 to 1 March 2021 were allocated to 1 of 3 groups according to the clinical symptoms and lesions (Group 1: bacteremia; Group 2: meningitis; Group 3: pleurisy). Taking Alere BinaxNow ${ }^{\circledR}$ Streptococcus pneumoniae (S. pneumoniae) antigen detection and blood culture as the gold standard, receiver operating characteristic (ROC) was used to establish the diagnostic value of mNGS.

Results: A total of 96 cases were enrolled in the study, comprising Group $1(n=65)$, Group 2 ( $n=17)$, and Group $3(n=14)$. The positive rate of mNGS test was $62.5 \%(n=60)$, and the total coincidence rate was $75.0 \%$. Delayed mNGS was found to have no significant effect on the 30-day survival rate; however, the species-specific read number (SSRN) of S. pneumoniae detected by mNGS in the early stage of the disease was higher, and it could significantly reduce the hospitalization days and costs $(\mathrm{P}<0.05)$.

Conclusions: The sensitivity and specificity of mNGS are high in the identification of S. pneumoniae in blood, cerebrospinal fluid, and pleural effusion samples, and the SSRN of S. pneumoniae is related to the interval from onset to sample collection. Early mNGS detection has no significant effect on the 30-day survival rate among children with IPD, but it can reduce hospitalization costs and duration.

Keywords: Metagenomic next-generation sequencing (mNGS); invasive pneumococcal disease (IPD); speciesspecific read number (SSRN); children

Submitted Oct 26, 2021. Accepted for publication Dec 15, 2021.

doi: $10.21037 / \mathrm{tp}-21-533$

View this article at: https://dx.doi.org/10.21037/tp-21-533

\footnotetext{
$\wedge$ ORCID: 0000-0002-0065-8465.
} 


\section{Introduction}

Streptococcus pneumoniae (S. pneumoniae), usually found in the human nasopharynx, is a very important pathogen of invasive respiratory infections which poses a serious threat to children around the world. The World Health Organization (WHO) estimates that about 800,000 children die of $S$. pneumoniae every year (1). Infections of sterile sites caused by $S$. pneumoniae (2) include bacteremia, sepsis, bacterial meningitis, osteomyelitis, pleurisy, and are collectively referred to as invasive pneumococcal disease (IPD). The WHO has found that IPD is one of the main causes of mortality and disability in children under 5 years old in recent years (3).

However, with the popularity of vaccines and abuse of broad-spectrum antibiotics in China and internationally, the epidemiology and drug sensitivity of $S$. pneumoniae have changed to some extent $(4,5)$, and the incidence of multi-drug resistant infections has also increased annually. It is difficult to identify the early stage of IPD infection in children, which leads to prolonged hospitalization, antimicrobial resistance, and increased medical costs (6). Therefore, early identification and targeted anti-infective treatment are key to improving the prognosis of children with IPD.

At present, the identification of $S$. pneumoniae mainly depends on traditional diagnostic techniques (such as culture, nucleic acid detection, and so on). Traditional culture methods have a lower positive rate and take a longer time than metagenomic next-generation sequencing (mNGS), an unbiased method, which can detect all pathogens in clinical samples. Currently, there has been no report on the application of mNGS in children with IPD in China or globally. Early diagnosis of IPD by mNGS and targeted anti-infective treatment are expected to reduce medical costs, shorten hospital stays, and thus improve prognosis without increasing the incidence of bacterial drug resistance. The purpose of this study was to evaluate the diagnostic sensitivity and specificity of mNGS for children with IPD, and investigate the application value of mNGS. We present the following article in accordance with the STARD reporting checklist (available at https://dx.doi. org/10.21037/tp-21-533).

\section{Methods}

\section{Participants and sample collection}

A retrospective review was conducted of pediatric cases with S. pneumoniae infections admitted to Hebei Children's Hospital from 1 January 2017 to 1 March 2021. The inclusion criteria were as follows: S. pneumoniae was isolated from normal sterile sites (blood, cerebrospinal fluid, pleural effusion) in hospitalized children. The exclusion criteria were as follows: non-IPD; incomplete medical records; and those who did not undergo mNGS examination.

The patients were divided into 3 groups according to the clinical symptoms and lesions: Group 1 was characterized by bacteremia; Group 2 by meningitis with nervous system symptoms; and Group 3 by pleurisy with respiratory symptoms. Blood samples were collected within $2 \mathrm{~h}$ after admission in Group 1; cerebrospinal fluid samples were collected by lumbar puncture in Group 2; and pleural fluid samples and deep sputum samples were collected in Group 3. All operations were carried out under strict aseptic conditions, and the samples were examined by traditional pathogen culture and antigen test. All children received mNGS examination, some of which the test timing was consistent with the traditional method (within 7 days after onset), while others underwent mNGS when routine examination returned negative or anti-infective treatment had failed for 1 week (more than 7 days after onset).

The clinical data, species-specific read number (SSRN) of $S$. pneumoniae, length of stay, hospitalization costs, and prognosis of the 3 groups were compared to explore the best time to conduct mNGS; the results of mNGS in different body fluid samples were compared with those of traditional etiological method to analyze the diagnostic efficiency of mNGS.

\section{Process of $m$ NGS}

Clinical information of all participants were sent together with samples to BGI Patho-Genesis Pharmaceutical Technology Co., Ltd. (Shenzhen, China), which is qualified for mNGS testing.

\section{Sample processing and DNA extraction}

Approximately 3-5 $\mathrm{mL}$ peripheral blood [ethylenediamine tetraacetic acid (EDTA) anticoagulant] was collected from all participants, and then $300 \mu \mathrm{L}$ plasma was obtained by highspeed centrifugation at $4{ }^{\circ} \mathrm{C}, 4,000 \mathrm{rpm}$, for $10 \mathrm{~min}$. Another $600 \mu \mathrm{L}$ body fluid (cerebrospinal fluid or pleural effusion) was taken from participants in Group 2 and Group 3. Glass beads and enzymes were blended well into each sample, and genomic DNA was extracted using TIANamp 
Micro DNA Kit (DP316, TIANGEN BIOTECH, Beijing, China) according to the manufacturer's instructions (7).

\section{DNA library preparation and sequencing}

We generated DNA fragments with sizes ranging from 200 to 300 bp using an Agilent 2100 Bioanalyzer (Agilent, Santa Clara, CA, USA), and formed a single-chain structure through cyclization. We then replicated the fragments using a rolling ring to form DNB nanospheres, which were loaded into the sequencing chip and sequenced using the BGISEQ-50/MGISEQ-2000 systems (BGI, China) (8).

\section{Data analysis}

Quality control was carried out after high-quality sequencing data had been obtained (9). According to the Burrows-Wheeler Aligner software (BWA; http://biobwa.sourceforge.net/), high-quality data consistent with the human reference genome sequence were screened and removed. After removing the low complexity reads, the remaining data were compared with 4 large microbial databases: special bacteria (6,350 species), fungi (1,064 species), viruses (4,945 species), and parasites (234 species). The sequence number that could match a certain pathogen was obtained, and the possible pathogens were judged according to the sequence number and other clinical tests. S. pneumoniae was considered potential pathogen when literature reported the pathogenicity or the score was as least two-fold greater than of that of any other microbe of the same type identified in the patients (10).

\section{Distinguish colonizing bacteria}

Introduction of negative controls (refer to the database): comparing test samples with negative control samples to identify species with no significant difference in reading; based on the historical detection: statistics on the frequency of microorganisms detected in the laboratory over a certain period, and the microorganisms detected at high frequencies as a background microbial database (colonizing strains).

\section{Verification of S. pneumoniae}

The decision was made according to the patient's history, physical examination, laboratory test results, imaging findings, and response to antibiotic treatment, as well as the experience and opinions of many clinicians.

When the pathogenicity was consistent with clinical manifestations, the Alere BinaxNOW ${ }^{\circledR}$ Streptococcus pneumoniae antigen test was verified (11), which has a moderate sensitivity and high specificity in Chinese (12).

\section{Statistical analysis}

The software SPSS 21.0 (IBM Corp., Armonk, NY, USA) was used for statistical analysis in this study. After examining the normality of distribution, continuous variables were represented as the mean \pm standard deviation or the median (minimum-maximum). Comparisons between 2 groups were analyzed with independent $t$-test or Mann-Whitney $\mathrm{U}$ test, where appropriate. Concerning the differences among the 3 groups, analysis of variance (ANOVA) or Kruskal-Wallis $\mathrm{H}$ tests were performed as appropriate. Receiver operating characteristic (ROC) was used to establish the diagnostic value of $\mathrm{mNGS}$. Statistical significance was set at $\mathrm{P}<0.05$.

\section{Ethical statement}

The study was conducted in accordance with the Declaration of Helsinki (as revised in 2013). This work was carried out with human research ethics approval from the Hebei Children's Hospital (No. 223). Written informed consents were provided by the legal guardians of enrolled children.

\section{Results}

\section{Patient characteristics and traditional pathogens}

A total of 96 children including 53 boys and 43 girls with IPD were enrolled in this study, with a median age of 19 months (range, 1-168 months). The most common manifestation was bacteremia $(\mathrm{n}=65)$, followed by meningitis $(\mathrm{n}=17)$, and pleurisy was the least common $(\mathrm{n}=14)$ (Figure 1). Among the participants, 17 cases had respiratory failure and received mechanical ventilation, 14 were complicated with septic shock, and 6 with cerebral hernia. Table 1 shows the data of gender, age, days from onset to visit, presence of fever, peripheral blood white blood cells (WBC), C-reactive protein (CRP), procalcitonin (PCT), and prognosis in each group.

Among the 96 cases with IPD enrolled in the study, the positive rate of culture method was $27.1 \%(\mathrm{n}=26)$, and the positive coincidence rate, negative coincidence rate, and total coincidence rate were $30.6 \%, 72.7 \%$, and $35.4 \%$, 
respectively.

\section{Detection efficiency of $\mathrm{mNGS}$}

The positive rate of mNGS test was $62.5 \%(\mathrm{n}=60)$, and the

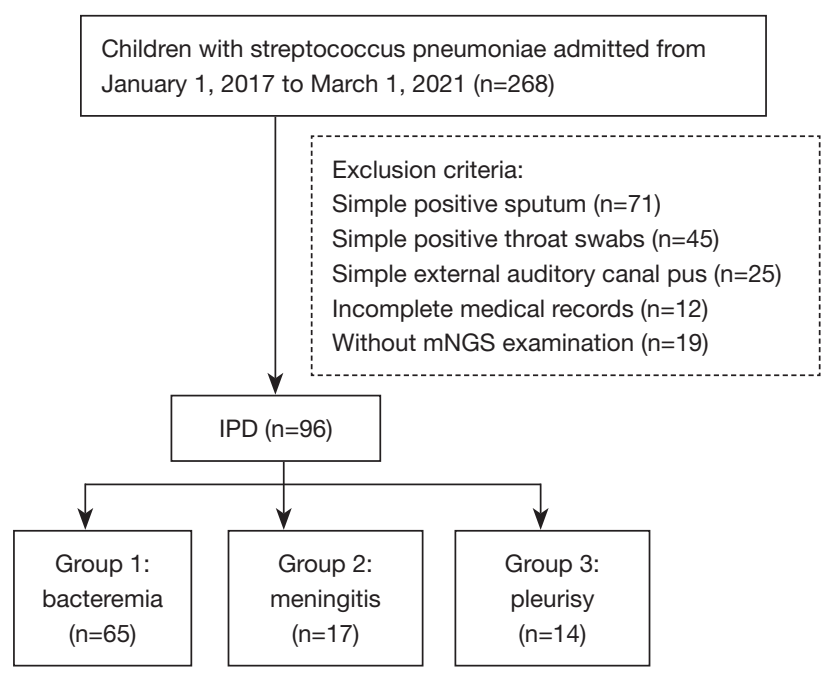

Figure 1 Flow chart of enrolled IPD children. IPD, invasive pneumococcal disease; mNGS, metagenomic next-generation sequencing. total coincidence rate was $75.0 \%$.

\section{Results of different groups}

A total of 65 children with IPD were included in group 1 . The ROC curve was performed (Figure 2A) to establish the diagnostic value of mNGS. When SSRN $\geq 75$ was considered to be positive, the value of the area under the curve (AUC) was the largest [0.743, 95\% confidence interval (CI): 0.585 to 0.901$]$. The sensitivity and specificity were $73.6 \%$ and $75 \%$, respectively, and the diagnostic coincidence rate was $73.8 \%$. The positive predictive value (PPV) and negative predictive value (NPV) were $92.3 \%$ and $39.1 \%$, respectively.

There were 17 and 14 children who were assigned into Group 2 and Group 3, respectively. Both groups were nonblood samples, so we combined them to draw the ROC (Figure 2B). The ROC curve showed that when SSRN was $\geq 15$, the AUC value of Group 2 and Group 3 was the highest $(0.704,95 \%$ CI: 0.431 to 0.977$)$, and the sensitivity, specificity, PPV, NPV, and diagnostic coincidence are summarized in Table 2.

The mNGS method had higher sensitivity and specificity for the identification of $S$. pneumoniae than traditional culture method, and the difference was statistically

Table 1 Clinical characteristics and laboratory tests of children with IPD

\begin{tabular}{lccc}
\hline Characteristics & Group 1 $(\mathrm{n}=65)$ & Group 2 $(\mathrm{n}=17)$ & Group $3(\mathrm{n}=14)$ \\
\hline Age $($ month), median (range) & $13(1$ to 168$)$ & $48(1$ to 132$)$ & $48(1$ to 108$)$ \\
Gender & & & \\
Male & $34(52.3 \%)$ & $11(64.7 \%)$ & $8(57.1 \%)$ \\
Female & $31(47.7 \%)$ & $6(35.3 \%)$ & $6(42.9 \%)$ \\
Fever & $57(87.7 \%)$ & $13(76.5 \%)$ & $12(85.7 \%)$ \\
Peripheral blood & & & $19 \pm 6$ \\
WBC $\left(\times 10^{6}\right)$ & $21 \pm 7$ & $19 \pm 6$ & $98 \pm 60$ \\
CRP (mg/L) & $94 \pm 56$ & $98 \pm 56$ & $67 \pm 30$ \\
PCT ( $\mu$ g/L) & $62 \pm 44$ & $67 \pm 30$ & $3(21.4 \%)$ \\
Respiratory failure & $10(15.4 \%)$ & $4(23.5 \%)$ & $1(7.1 \%)$ \\
Sepsis shock & $11(16.9 \%)$ & $2(11.8 \%)$ & 0 \\
Cerebral hernia & $2(3.1 \%)$ & $4(23.5 \%)$ & $4.9 \pm 2.8$ \\
Days from onset to visit (d) & $4.2 \pm 2.6$ & $4.9 \pm 2.8$ & $12(85.7 \%)$ \\
Survival over 30 days & $59(90.8 \%)$ & $16(94.1 \%)$ & \\
\hline
\end{tabular}

IPD, invasive pneumococcal disease; WBC, white blood cell; CRP, C-reactive protein; PCT, procalcitonin. 

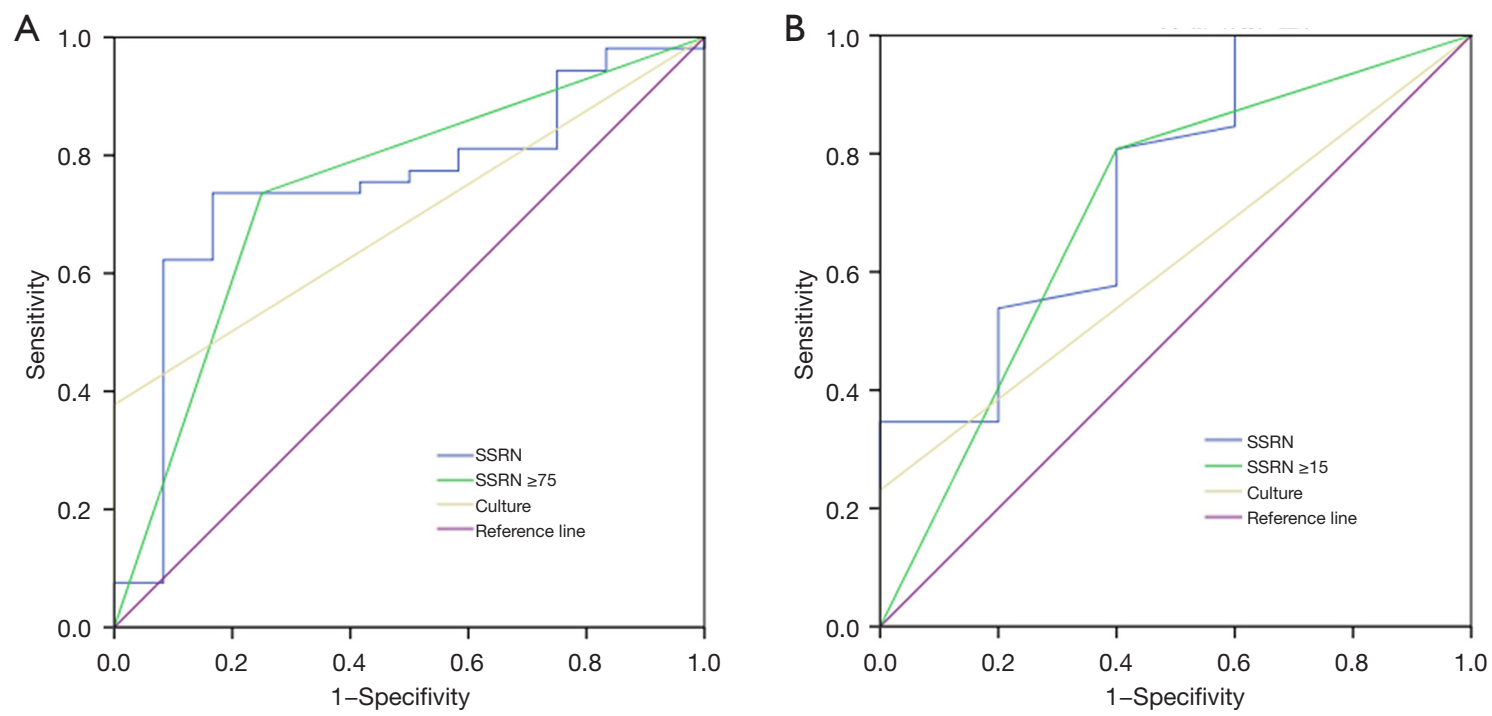

Figure 2 ROC curves. The diagnostic performance of mNGS in Group 1 (A) and Group 2, 3 (B). SSRN, species-specific read number; ROC, receiver operating characteristic; mNGS, metagenomic next-generation sequencing.

Table 2 Comparison of pathogen detection results

\begin{tabular}{|c|c|c|c|c|c|c|c|c|}
\hline Diagnostic performance & \multicolumn{4}{|c|}{ mNGS } & \multicolumn{4}{|c|}{ Culture } \\
\hline Positive cases & 39 & 11 & 10 & 60 & 20 & 2 & 4 & 26 \\
\hline Sensitivity (\%) & 73.6 & 73.3 & 90.9 & 75.9 & 37.7 & 13.3 & 36.3 & 30.6 \\
\hline Specificity (\%) & 75 & 100 & 100 & 70.6 & 100 & 100 & 100 & 100 \\
\hline NPV (\%) & 39.1 & 33.3 & 25.0 & 38.7 & 26.7 & 13.3 & 30.0 & 24.3 \\
\hline DCR (\%) & 73.8 & 76.5 & 78.6 & 75.0 & 49.2 & 23.5 & 50.0 & 44.8 \\
\hline
\end{tabular}

mNGS, metagenomic next-generation sequencing; PPV, positive predictive value; NPV, negative predictive value; DCR, Diagnostic coincidence rate.

significant $(\mathrm{P}<0.05)$.

\section{Effect of detection timing on SSRN}

The effect of mNGS detection timing on SSRN of $S$. pneumoniae in children with IPD is compiled in Table 3. The earlier the sample was collected, the larger the SSRN of $S$. pneumoniae was, and the difference was statistically significant $(\mathrm{P}<0.05)$.

\section{Effect of detection timing on prognosis}

Early detection of mNGS had no significant effect on the 30-day survival rate (Table 4), the survival curve of mNGS positive group crossed with the negative group (Figure 3). A total of 9 children with IPD died in this study, among whom septic shock was the most common cause.

However, we found that among the surviving children who underwent mNGS testing more than 7 days after onset, 4 cases developed serious complications requiring surgical intervention (including 1 case of osteomyelitis for surgical treatment, 1 case of hydrocephalus for drainage, 1 case of brain atrophy that may require long-term rehabilitation treatment, and 1 case of lung abscess for resection of necrotic lung lesions). No serious complications occurred in the surviving children who underwent mNGS within 
Table 3 Comparison of SSRN of S. pneumoniae with different mNGS detection timings

\begin{tabular}{lcccc}
\hline \multirow{2}{*}{ Detection timing } & \multicolumn{3}{c}{ SSRN } & \\
\cline { 2 - 4 } & Group 1 & Group 2 & Group 3 & \\
\hline Within 7 d & $165 \pm 101$ & $49 \pm 33$ & $84 \pm 29$ & 0.000 \\
later than 7 d & $47 \pm 30$ & $13 \pm 7$ & $24 \pm 13$ & 0.021 \\
Z & -3.858 & -2.062 & -3.000 & \\
P value & 0.000 & 0.037 & 0.003 & \\
\hline
\end{tabular}

SSRN, species-specific read number; mNGS, metagenomic next-generation sequencing.

Table 4 Comparison of survival over 30 days with different mNGS detection timings

\begin{tabular}{lccc}
\hline \multirow{2}{*}{$\begin{array}{l}\text { Detection } \\
\text { timing }\end{array}$} & \multicolumn{3}{c}{ Survival over 30 days } \\
\cline { 2 - 4 } & Group 1 $(\mathrm{n}=65)$ & Group 2 $(\mathrm{n}=17)$ & Group 3 $(\mathrm{n}=14)$ \\
\hline Within 7 d & $50 / 54$ & $10 / 11$ & $8 / 9$ \\
Later than 7 d & $9 / 11$ & $1 / 1$ & $4 / 5$ \\
$P$ value & 0.219 & 0.478 & 1.000 \\
\hline
\end{tabular}

mNGS, metagenomic next-generation sequencing.

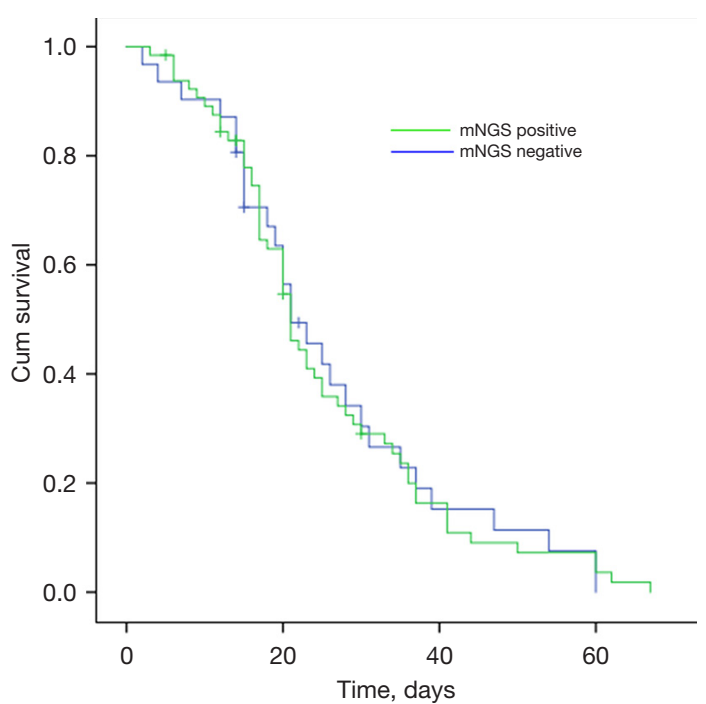

Figure 3 Survival curve of mNGS positive group and negative group. mNGS, metagenomic next-generation sequencing.
7 days after onset. In addition, compared with delayed mNGS detection, children with IPD who received early examination ( $<7$ days) had lower hospitalization costs and length of stay, and the difference was statistically significant $(\mathrm{P}<0.05)$ (Table 5).

\section{Discussion}

Children are generally susceptible to $S$. pneumoniae. Although 13-valent pneumoniae conjugate vaccine (PCV13) has been formally introduced in China since June 2017, it has not been included in the National Immunization Plan, and variant serotypes of $S$. pneumoniae emerge incessantly, which can lead to ineffectiveness of existing vaccines (13). Moreover, it has been reported that the total carrying rate of $S$. pneumoniae in children is up to $17.8 \%$ (14). Patients with IPD are at risk of insomnia, hearing impairment, language and mental developmental delays (15-17), and even septic shock and death in severe cases (18). For children, delayed treatment of IPD can increase the risk of septic shock and mechanical ventilation by 4.58 times and 2.7 times, respectively, and the risk of death by nearly 40 times (19). The purpose of our study was to explore the timing and value of the application of mNGS and to find a way to improve the prognosis for children with IPD through comparison with traditional pathogen detection.

The traditional pathogen detection methods for $S$. pneumoniae mainly include PCR, serology and culture. Previous studies have mostly used polymerase chain reaction (PCR) to verify specific pathogens; however, the cell wall of $S$. pneumoniae is difficult to dissolve, and human DNA may inhibit $S$. pneumoniae in blood samples. Therefore, the value of PCR for S. pneumoniae in blood samples is limited. The Alere BinaxNOW ${ }^{\circledR}$ Streptococcus pneumoniae antigen test is a serological method for $S$. pneumoniae with high specificity but moderate sensitivity, which couldn't meet clinical needs. Therefore, it was used as a method to verify $S$. pneumoniae in our study. Our study has found that mNGS has higher sensitivity and specificity in the diagnosis of pathogens in children with IPD than culture. Previous studies have reported that the sensitivity of mNGS for bacterial identification was $79.2 \%$, and the specificity was $90.6 \%$ (20). In our study, it was found that the sensitivity and specificity 
Table 5 Comparison of hospitalization days and costs with different timings

\begin{tabular}{|c|c|c|c|c|c|c|c|c|c|c|}
\hline Detection timing & \multicolumn{3}{|c|}{ Hospitalization days (d) } & $\mathrm{F}$ & $P$ value & \multicolumn{3}{|c|}{ Hospitalization costs $(10,000 \mathrm{RMB})$} & $\mathrm{F}$ & $P$ value \\
\hline Within $7 \mathrm{~d}$ & $30 \pm 16$ & $22 \pm 10$ & $13 \pm 6$ & 4.393 & 0.018 & $8.2 \pm 3.4$ & $7.9 \pm 3.0$ & $5.1 \pm 1.9$ & 2.329 & 0.109 \\
\hline Later than $7 \mathrm{~d}$ & $23 \pm 15$ & $21 \pm 6$ & $14 \pm 3$ & 0.523 & 0.597 & $10.4 \pm 3.5$ & $12.3 \pm 2.7$ & $9.7 \pm 4.4$ & 1.069 & 0.352 \\
\hline $\mathrm{t}$ & -3.593 & -2.161 & -3.457 & & & -2.602 & -3.159 & -2.351 & & \\
\hline
\end{tabular}

of mNGS are higher than those of traditional pathogen detection, whether in children with IPD characterized by bacteraemia or in children characterized by meningitis and pleurisy. The reasons are as follows: (I) S. pneumoniae is not only a traditional extracellular pathogen, but it can also survive in the unique vacuoles of the host cell, so as to avoid degradation by acid lysosome, regulate the expression of its virulence gene to adapt to the intracellular environment, evade immune surveillance, and spread in the host (21), thus affecting the results of traditional culture; (II) the use of antibiotics can destroy the pathogen, but the DNA of the pathogen survives for a long time in plasma, and the mNGS detection is aimed at the nucleic acid sequence of the pathogen, not at the pathogen itself. Therefore, the use of antibiotics has little effect on the results of mNGS detection, while the traditional culture methods are greatly affected by the use of antibiotics $(22,23)$. If the results of mNGS and traditional pathogen detection can be seamlessly combined, the sensitivity and specificity of pathogen identification of $S$. pneumoniae will be more ideal (24). In general, mNGS has high sensitivity and specificity, and is less affected by antibiotics and host cell expression. In addition, some of our subjects had low immune function and were simultaneously infected with pneumocystis or aspergillus after chemotherapy. For these children, the undifferentiated detection of pathogens by mNGS was incomparable to traditional detection methods.

Our study also found that the SSRN of S. pneumoniae tested within 7 days is significantly higher than that tested more than 7 days after onset, which indicates that the SSRN of $S$. pneumoniae detected by mNGS is related to the timing of sample collection. The earlier the mNGS test is carried out, the greater the possibility of positive results. In the early stages, $S$. pneumoniae increase intracellular invasion and survival by using various virulence factors such as pneumolysin (PLY), pneumococcal surface protein A (PSPA), pneumococcal adhesion and virulence protein
B (PavB), bacterial hair-1 adhesin RrgA, pyruvate oxidase (SpxB), and metalloproteinase (ZMPB). The clearance of the host significantly decreased, at this time line the sequence number of pathogenic nucleic acid is high. In contrast, with the application of antimicrobial drugs and the gradual restoration of immune function, the $\mathrm{CD} 4+\mathrm{T}$ cell reaction is enlarged, macrophages are induced, and the immune response is continuously amplified to activate neutrophils and directly kill pathogens, resulting in the gradual reduction of the nucleic acid sequence of pathogens (25). So the SSRN of pathogens decreases with the use of antibiotics and the improvement of the disease, which is consistent with the previous studies $(26,27)$.

In this study, mNGS was performed in blood, cerebrospinal fluid, and pleural effusion samples of children with IPD. For different body fluid samples, the optimal SSRN of $S$. pneumoniae is different, because so far there is no unified standard for the identification of a single pathogen by mNGS, and the DNA extraction methods and human host sequence contents of different samples are different. Therefore, further research is yet to be done on the optimal SSRN for specific pathogens.

We found that delayed mNGS testing has no significant effect on the survival rate of children with IPD, but increases the hospitalization costs and days $(\mathrm{P}<0.05)$. In addition, surviving children who experience delayed mNGS testing have a higher rate of serious complications. This may be related to the severe infection and infiltration of our participants. Children who do not undergo early mNGS testing are more likely to be given broad-spectrum antimicrobials empirically, which not only increases the hospitalization costs, but also enhances the chance of adverse drug reactions and drug resistance, which, in turn, can lead to the prolongation of hospital stay. After the pathogen is identified by mNGS detection, targeted antiinfective treatment can be given early, which is in line with the process of evidence-based medicine, and better 
prognosis can be obtained. Therefore, early pathogen identification and timely and effective treatment for IPD children can reduce the hospitalization costs and improve the prognosis.

There were several limitations in our study, though. First, the study was retrospective and the number of cases was too small, which were likely to cause some deviations in the results, it needs to be further confirmed by a large sample and multi-center clinical trials. Second, there are no uniform criteria for the distinction between colonizing bacteria and pathogenic bacteria by mNGS, the results must be interpreted in a clinical context. Finally, for the diagnostic criteria of mNGS for a single pathogen, different researchers have reported different values based on coverage, relative abundance of pathogens, or unique readings of pathogens, but there is still no unified standard $(10,28)$.

\section{Conclusions}

In this study, the application value of mNGS in children with IPD was analyzed for the first time. Compared with traditional methods, mNGS has advantages in detecting $S$. pneumoniae: (I) the mNGS method has high sensitivity and specificity for the identification of $S$. pneumoniae in blood, cerebrospinal fluid, and pleural effusion samples; (II) it is less affected by antibiotics and expression of host cells; (III) it can be used for simultaneous infection of multiple pathogens. The SSRN of S. pneumoniae is related to the interval from onset to sample collection. Delayed sample collection can lead to decreased SSRN in children with IPD. Early mNGS detection has no significant effect on the 30-day survival rate of children with IPD, but can reduce the hospitalization costs and duration of stay.

\section{Acknowledgments}

Funding: None.

\section{Footnote}

Reporting Checklist: The authors have completed the STARD reporting checklist. Available at https://dx.doi. org/10.21037/tp-21-533

Data Sharing Statement: Available at https://dx.doi. org/10.21037/tp-21-533

Conflicts of Interest: All authors have completed the ICMJE uniform disclosure form (available at https://dx.doi. org/10.21037/tp-21-533). The authors have no conflicts of interest to declare.

Ethical Statement: The authors are accountable for all aspects of the work in ensuring that questions related to the accuracy or integrity of any part of the work are appropriately investigated and resolved. The study was conducted in accordance with the Declaration of Helsinki (as revised in 2013). This work was carried out with human research ethics approval from the Hebei Children's Hospital (No. 223). Written informed consents were provided by the legal guardians of enrolled children.

Open Access Statement: This is an Open Access article distributed in accordance with the Creative Commons Attribution-NonCommercial-NoDerivs 4.0 International License (CC BY-NC-ND 4.0), which permits the noncommercial replication and distribution of the article with the strict proviso that no changes or edits are made and the original work is properly cited (including links to both the formal publication through the relevant DOI and the license). See: https://creativecommons.org/licenses/by-nc-nd/4.0/.

\section{References}

1. World Health Organization. Pneumococcal conjugate vaccines in infants and children under 5 years of age: WHO position paper - February 2019. Wklyepidemiol Rec 2019;94:85-104.

2. Gerlini A, Colomba L, Furi L, et al. The role of host and microbial factors in the pathogenesis of pneumococcal bacteraemia arising from a single bacterial cell bottleneck. PLoS Pathog 2014;10:e1004026.

3. Wahl B, O'Brien KL, Greenbaum A, et al. Burden of Streptococcus pneumoniae and Haemophilus influenzae type $b$ disease in children in the era of conjugate vaccines: global, regional, and national estimates for 2000-15. Lancet Glob Health 2018;6:e744-57.

4. Wijayasri S, Hillier K, Lim GH, et al. The shifting epidemiology and serotype distribution of invasive pneumococcal disease in Ontario, Canada, 2007-2017. PLoS One 2019;14:e0226353.

5. Huang YC, Lin CF, Ting PJ, et al. Respiratory pathogens Some altered antibiotic susceptibility after implementation of pneumococcus vaccine and antibiotic control strategies. J Microbiol Immunol Infect 2020;53:682-9.

6. Llor C, Bjerrum L. Antimicrobial resistance: risk 
associated with antibiotic overuse and initiatives to reduce the problem. Ther Adv Drug Saf 2014;5:229-41.

7. Long Y, Zhang Y, Gong Y, et al. Diagnosis of Sepsis with Cell-free DNA by Next-Generation Sequencing Technology in ICU Patients. Arch Med Res 2016;47:365-71.

8. Jeon YJ, Zhou Y, Li Y, et al. The feasibility study of non-invasive fetal trisomy 18 and 21 detection with semiconductor sequencing platform. PLoS One 2014;9:e110240.

9. Li H, Durbin R. Fast and accurate short read alignment with Burrows-Wheeler transform. Bioinformatics 2009;25:1754-60.

10. Langelier C, Zinter MS, Kalantar K, et al. Metagenomic Sequencing Detects Respiratory Pathogens in Hematopoietic Cellular Transplant Patients. Am J Respir Crit Care Med 2018;197:524-8.

11. Selickman J, Paxos M, File TM Jr, et al. Performance measure of urinary antigen in patients with Streptococcus pneumoniae bacteremia. Diagn Microbiol Infect Dis 2010;67:129-33.

12. Zhou F, Gu L, Qu JX, et al. Evaluating the utility of Binax NOW Streptococcus pneumoniae urinary antigen test in adults with community acquired pneumonia in China. Clin Respir J 2018;12:425-32.

13. Principi N, Esposito S. Pneumococcal Disease Prevention: Are We on the Right Track? Vaccines (Basel) 2021;9:305.

14. Ceyhan M, Karadag-Oncel E, Hascelik G, et al. Nasopharyngeal carriage of Streptococcus pneumoniae in healthy children aged less than five years. Vaccine 2021;39:2041-7.

15. Korona-Glowniak I, Zychowski P, Siwiec R, et al. Resistant Streptococcus pneumoniae strains in children with acute otitis media- high risk of persistent colonization after treatment. BMC Infect Dis 2018;18:478.

16. Chen KJ, Chong YJ, Sun MH, et al. Streptococcus pneumoniae endophthalmitis: clinical settings, antibiotic susceptibility, and visual outcomes. Sci Rep 2021;11:6195.

17. Hailegiyorgis TT, Sarhie WD, Workie HM. Isolation and antimicrobial drug susceptibility pattern of bacterial pathogens from pediatric patients with otitis media in selected health institutions, Addis Ababa, Ethiopia: a prospective cross-sectional study. BMC Ear Nose Throat Disord 2018;18:8.

18. Asner SA, Agyeman PKA, Gradoux E, et al. Burden of Streptococcus pneumoniae Sepsis in Children After Introduction of Pneumococcal Conjugate Vaccines: A Prospective Population-based Cohort Study. Clin Infect Dis 2019;69:1574-80.
19. Li Q, Cheng J, Wu Y, et al. Effects of Delayed Antibiotic Therapy on Outcomes in Children with Streptococcus pneumoniae Sepsis. Antimicrob Agents Chemother 2019;63:e00623-19.

20. Gu W, Deng X, Lee M, et al. Rapid pathogen detection by metagenomic next-generation sequencing of infected body fluids. Nat Med 2021;27:115-24.

21. Subramanian K, Henriques-Normark B, Normark S. Emerging concepts in the pathogenesis of the Streptococcus pneumoniae: From nasopharyngeal colonizer to intracellular pathogen. Cell Microbiol 2019;21:e13077.

22. Gosiewski T, Ludwig-Galezowska AH, Huminska K, et al. Comprehensive detection and identification of bacterial DNA in the blood of patients with sepsis and healthy volunteers using next-generation sequencing method - the observation of DNAemia. Eur J Clin Microbiol Infect Dis 2017;36:329-36.

23. Rhodes J, Hyder JA, Peruski LF, et al. Antibiotic use in Thailand: quantifying impact on blood culture yield and estimates of pneumococcal bacteremia incidence. Am J Trop Med Hyg 2010;83:301-6.

24. Xing XW, Zhang JT, Ma YB, et al. Metagenomic NextGeneration Sequencing for Diagnosis of Infectious Encephalitis and Meningitis: A Large, Prospective Case Series of 213 Patients. Front Cell Infect Microbiol 2020;10:88.

25. Liu Chao, Han Zhiying. Progress of invasive pneumococcal disease in children. J Int J Pediatr 2021;48:262-6.

26. Guo LY, Li YJ, Liu LL, et al. Detection of pediatric bacterial meningitis pathogens from cerebrospinal fluid by next-generation sequencing technology. J Infect 2019;78:323-37.

27. Ai JW, Zhang HC, Cui P, et al. Dynamic and direct pathogen load surveillance to monitor disease progression and therapeutic efficacy in central nervous system infection using a novel semi-quantitive sequencing platform. J Infect 2018;76:307-10.

28. Li H, Gao H, Meng H, et al. Detection of Pulmonary Infectious Pathogens From Lung Biopsy Tissues by Metagenomic Next-Generation Sequencing. Front Cell Infect Microbiol 2018;8:205.

(English Language Editor: J. Jones)

Cite this article as: Guo F, Kang L, Xu M. The application value of metagenomic next-generation sequencing in children with invasive pneumococcal disease. Transl Pediatr 2021;10(12):3282-3290. doi: 10.21037/tp-21-533 